Frédéric Grillot, Heming Huang, Lyu-Chih Lin, Chih-Ying Chen, Dejan Arsenijevic, Dieter Bimberg, Fan-Yi Lin

\title{
Recent advances in InAs/GaAs quantum dot lasers with short optical feedback
}

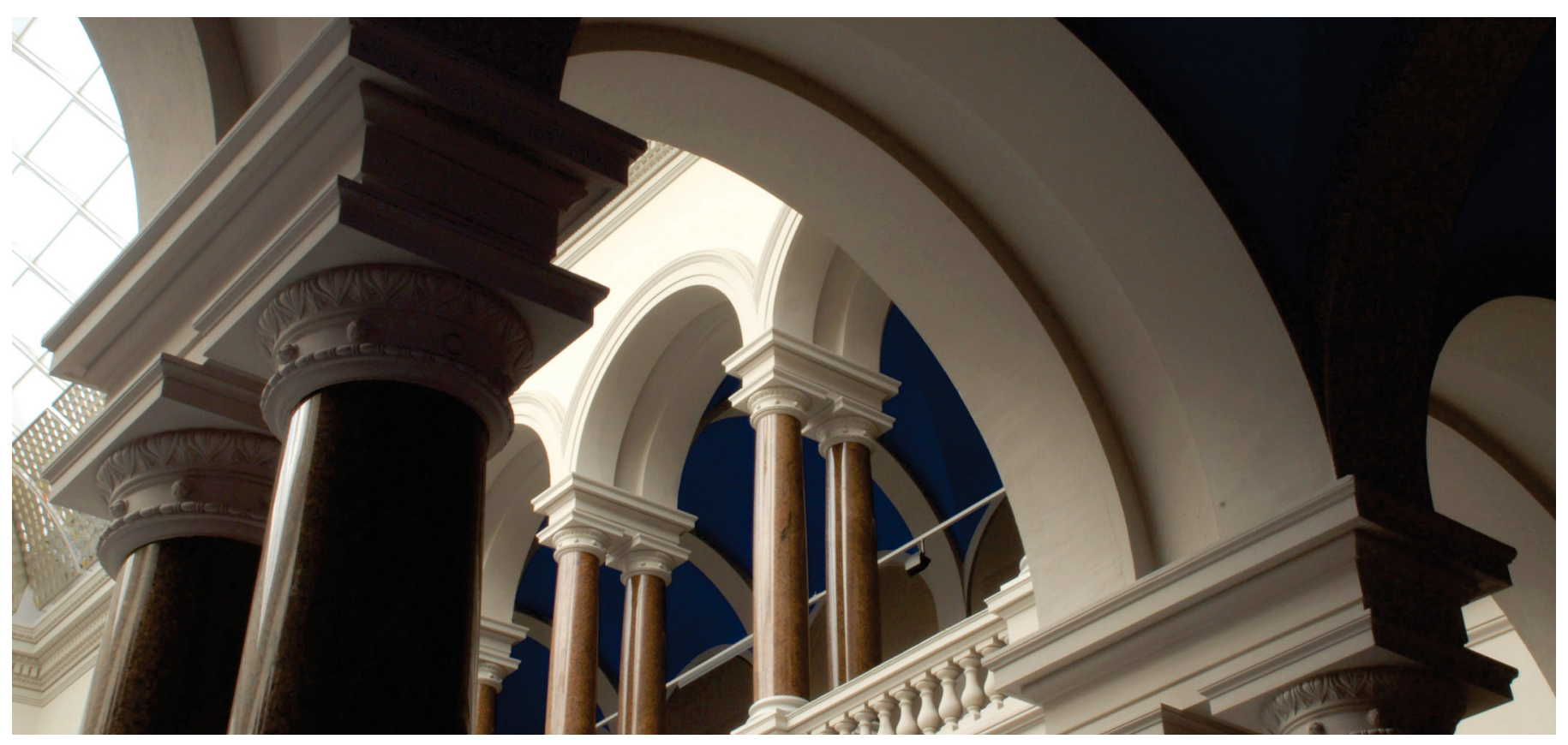

Grillot, F., Huang, H., Lin, L.-C., Chen, C.-Y., Arsenijevic, D., Bimberg, D. \& Lin, F.-Y. (2018). Recent advances in InAs/GaAs quantum dot lasers with short optical feedback. Proc. SPIE 10682, Semiconductor Lasers and Laser Dynamics VIII, 106820Y (9 May 2018). https://doi.org/10.1117/12.2314708. 


\title{
Recent advances in InAs/GaAs quantum dot lasers with short optical feedback
}

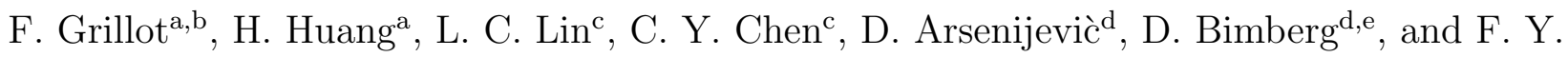 \\ $\operatorname{Lin}^{\mathrm{c}}$ \\ ${ }^{a}$ LTCI, Télécom ParisTech, Université Paris-Saclay, 46 rue Barrault, Paris, France \\ ${ }^{\mathrm{b}}$ Center for High Technology Materials, University of New-Mexico, Albuquerque, USA \\ 'Institute of Photonics Technologies, Department of Electrical Engineering, National Tsing \\ Hua University, Hsinchu 300, Taiwan \\ ${ }^{\mathrm{d} I n s t i t u t ~ f u ̈ r ~ F e s t k o ̈ r p e r p h y s i k, ~ T e c h n i s c h e ~ U n i v e r s i t a ̈ t ~ B e r l i n, ~ B e r l i n ~ 10623, ~ G e r m a n y ~}$ \\ ${ }^{\mathrm{e}}$ Chinese-German Green Photonics Center at the Chinese Academy of Sciences, CIOMP, \\ Changchun, China
}

\begin{abstract}
The optical feedback dynamics of two multimode InAs/GaAs quantum dot lasers emitting exclusively on sole ground or excited lasing states is investigated under the short delay configuration. Although the two lasers are made from the same active medium, their responses to the external perturbation are found not much alike. By varying the feedback parameters, various periodic and chaotic oscillatory states are unveiled. The ground state laser is found to be much more resistant to optical feedback, benefiting from its strong relaxation oscillation damping. In contrast, the excited state laser can easily be driven into very complex dynamics. While the ground state laser is of importance for the development of isolator-free transmitters, the excited one is essential for applications taking advantages of chaos such as chaos lidar, chaos radar, and random number generation.
\end{abstract}

Keywords: Quantum dot lasers, optical feedback, dynamical states

\section{INTRODUCTION}

The nonlinear dynamics of semiconductor lasers operating under external optical feedback has been widely studied in the literature. ${ }^{1}$ Indeed, by controlling the feedback strength and the external cavity length, multiple complex dynamical states and their routes to chaos can be observed. ${ }^{1}$ Compared to quantum well (QW) lasers, significant breakthroughs have been achieved by using quantum dots (QD) as gain media ${ }^{2}{ }^{3}$ Their inherent properties allow producing energy- and cost-efficient devices with outstanding temperature stability, low threshold current, ultrafast gain dynamics, and low amplified spontaneous emission. ${ }^{3}$ A low threshold current density and high internal quantum efficiency results in a reduced amount of dissipated heat. Even at high operation temperatures, temperature insensitive threshold currents have been observed for p-doped devices. ${ }^{4}$ Most advantages of QD lasers have been demonstrated for InAs/GaAs QD lasers operating at $1310 \mathrm{~nm}$, hence mostly targeting short communication links such as metro and access networks. QD lasers usually show three possible regimes of lasing operation, depending on the bias conditions: (i) ground state (GS) lasing; (ii) dual state emission showing an interplay dynamic between the GS and the first excited state (ES), and (iii) ES lasing ${ }^{5}{ }^{6}$ While feedback dynamics of QD lasers has been widely studied in particular in the context of the dual-state dynamics, ${ }^{7}$ none of this work focused on the feedback dynamics of QD devices emitting exclusively on single lasing states. Here, we report for the first time on the feedback dynamics of two InAs/GaAs QD Fabry-Perot (FP) lasers sharing the same active regions but emitting exclusively on either the GS or the ES. In other words, the QD lasers under study do not exhibit the GS-ES interplay dynamics where ES and GS lasing take place simultaneously. By varying the feedback strength under the short delay configuration, multiple dynamical states such as periodic $(\mathrm{P})$, regular pulse package (RPP), quasi-chaos pulse package (QCPP), and chaotic (C) states are unveiled. The

Further author information: (Send correspondence to F.G.)

F.G.: E-mail: grillot@telecom-paristech.fr 
GS laser is found to be rather insensitive to optical feedback due to the large damping factor. In contrast, the ES laser can be driven more easily into complex dynamical regimes including chaotic states. We believe that these results are of primary importance for utilizing the nonlinear dynamics for ultrafast devices in particular for the development of isolator-free transmitters, integrated self-pulsating devices and other applications using diode laser chaos. ${ }^{8}$

\section{LASER STRUCTURE AND EXPERIMENTAL SETUP}

The dynamical characteristics of the two FP multimode QD lasers, one emitting exclusively on the GS and one on the ES, are investigated and compared under different operation and feedback conditions. For both lasers, their active regions comprise $10 \mathrm{InAs}$ dot sheets grown in InGaAs QWs by molecular beam epitaxy (MBE) with a dot-in-well structure. The dot densities are around $3 \sim 5 \times 10^{10} \mathrm{~cm}^{-2}$ per layer and their lateral extensions approach $30 \mathrm{~nm}$. Both laser types have internal cavities of $1 \mathrm{~mm}$ long and $2 \mu \mathrm{m}$ waveguides etched through the active area. Lasers are left as-cleaved and cavity lengths are both $1 \mathrm{~mm}$ long while the ridge waveguide (RWG) etched through the active region is $2 \mu \mathrm{m}$ wide. For the GS laser, the threshold current $I_{t h}$ is of $16.5 \mathrm{~mA}$ and the external efficiency is $21 \%$. For the ES one, the threshold current $I_{t h}$ is of about $88 \mathrm{~mA}$ and the external efficiency is $11 \%$. Figures 1(a) and 1(b) display the two optical spectra taken at 1.5 times the threshold. The first FP laser (in red) emits at $\sim 1300 \mathrm{~nm}$ on the sole GS transition whereas the second (in blue) is at $\sim 1230 \mathrm{~nm}$ on the sole ES transition. The insets highlight the center of the emission of both lasers respectively, where the ES one exhibits a modulated optical spectral envelope in contrast to the GS laser. ${ }^{9}$ In this work, the ES selection was obtained by exploiting the natural wavelength dispersion of the photoluminescence peak across the entire wafer. ${ }^{9}$ Figure

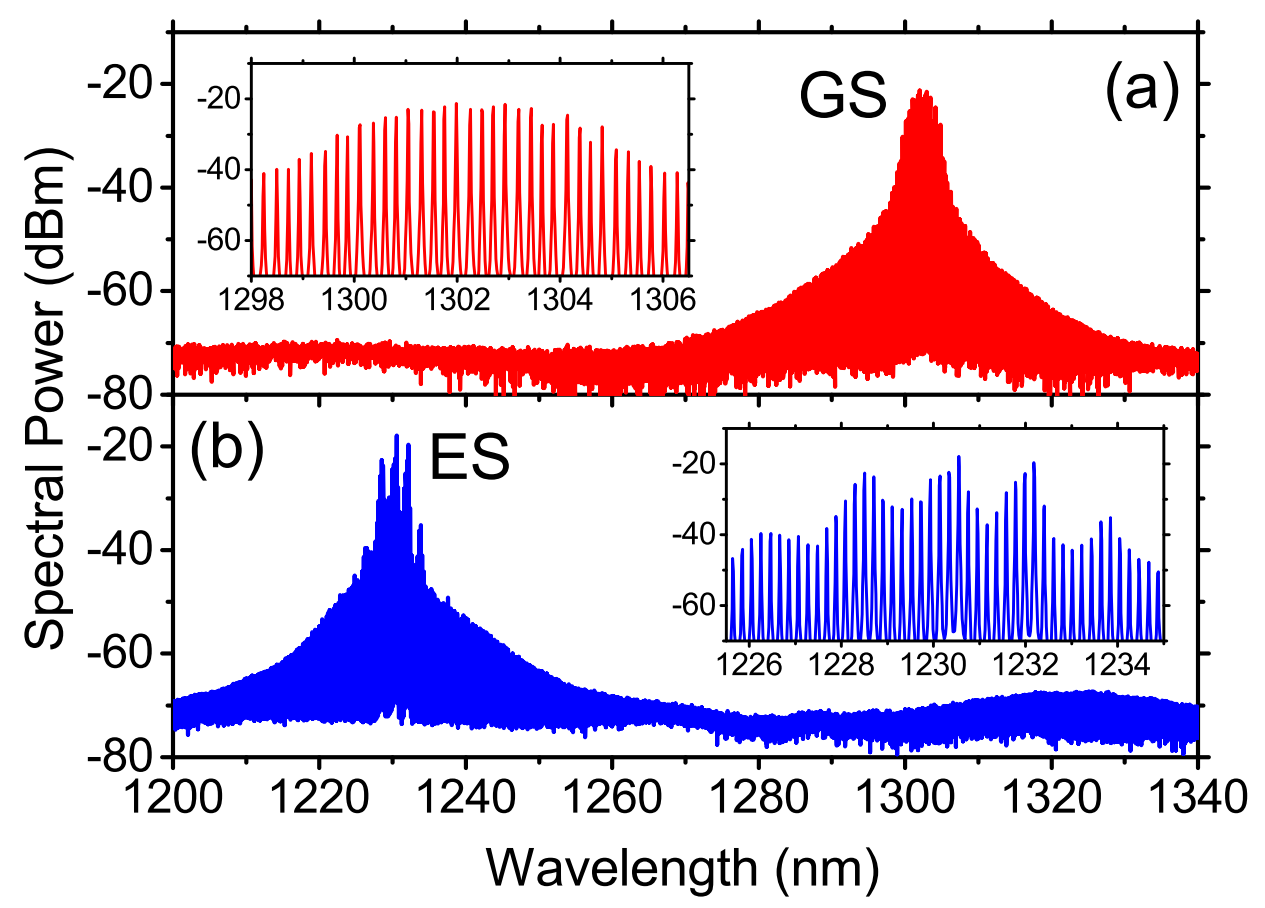

Figure 1. Optical spectra of the (a) GS and (b) ES lasers measured at 1.5 times the threshold under free-running condition (no feedback applied).

2 shows the schematic setup of the QD laser subject to optical feedback. The output of the laser towards the left is fed back to the laser cavity through a partially reflecting mirror to form an external cavity with length $L_{\text {ext }}$ with a minimum cavity length of $2 \mathrm{~cm}$. The ratio between the frequency of the external cavity $f_{\text {ext }}$ and the relaxation oscillation frequency $f_{\mathrm{RO}}$ is such as $f_{\mathrm{ext}} / f_{\mathrm{RO}}>1$ meaning that the short cavity regime is investigated in this paper. A variable optical attenuator is used to adjust the feedback strength $\xi_{\mathrm{f}}$ defined as the ratio of the feedback field to the laser output field. The optical signals are analyzed by an optical spectrum analyzer and 
the optical power is measured by a power meter. The electrical signals are detected by two identical high-speed photodetectors and analyzed by an electrical spectrum analyzer and a real-time oscilloscope.

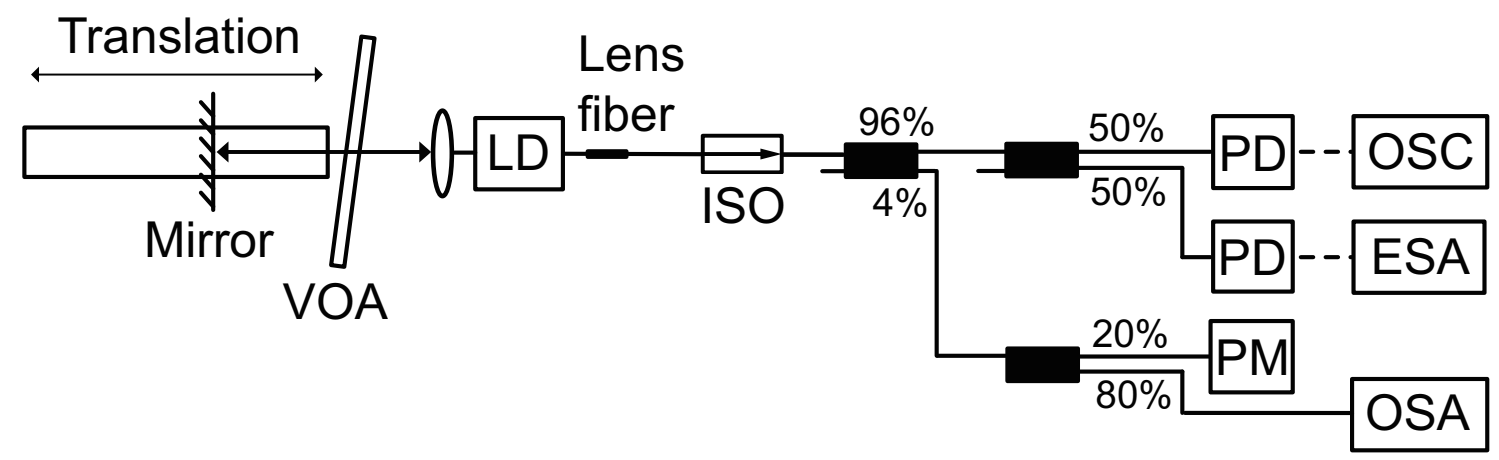

Figure 2. Experimental setup of a QD laser subject to optical feedback. LD: QD laser; ISO: isolator; PD: high-speed photodetector; ESA: electrical spectrum analyzer; OSA: optical spectrum analyzer; OSC: oscilloscope; PM: power meter.

\section{RESULTS AND DISCUSSION}

Figure 3 shows the time series and the corresponding power spectra of the dynamical states measured at 1.7 times the threshold for the GS laser. At $L_{\text {ext }}=30 \mathrm{~mm}$, a RPP state is demonstrated in Figures 3(a) and 3(b) for $\xi_{\mathrm{f}}=0.826$. The RPP regime is a typical complex dynamics arising under optical feedback, which manifests as regular pulsations in the output power. ${ }^{10}$ It occurs in the very short cavity regime meaning that a very small
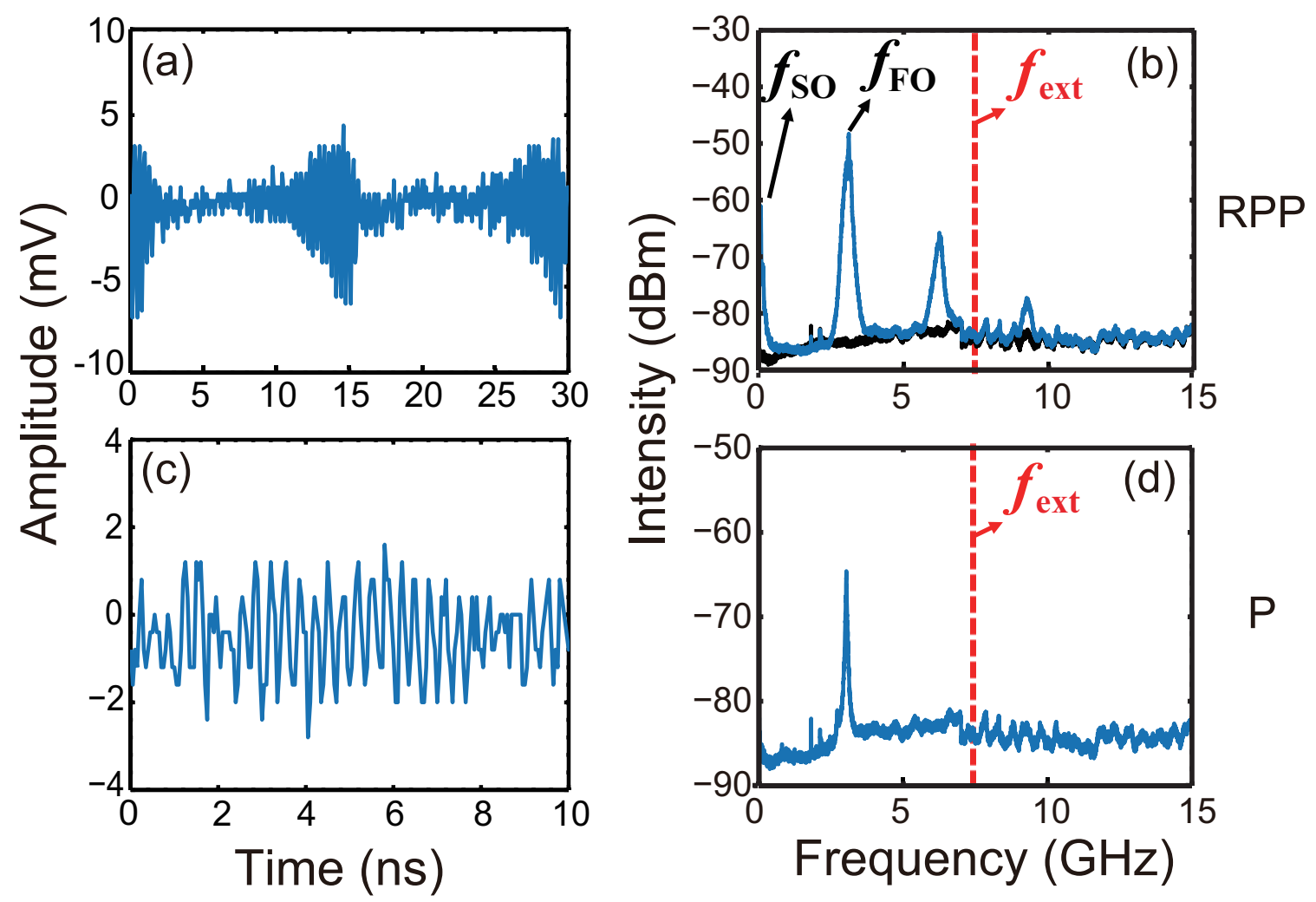

Figure 3. Time series and corresponding power spectra of the dynamical states measured at 1.7 times the threshold for the GS laser with $\left(\xi_{\mathrm{f}}, L_{\text {ext }}\right)=(\mathrm{a})(\mathrm{b})(0.826,30 \mathrm{~mm})$, (c)(d) $(0.731,30 \mathrm{~mm})$. Red-dashed lines depict the external cavity frequency $f_{\text {ext }}$ and black curves are the spectra without feedback for reference. 
number of modes is involved. Unlike the low-frequency fluctuation regime taking place when $f_{\text {ext }}<<f_{R O}$, the path in the phase space for the RPP regime is one direction only, which means that the crisis is no longer present, and the trajectory always visits the same external cavity modes leading to the birth of fast and regular oscillations at $f_{\text {ext }}$. Different from the RPP state described in ${ }^{10}$ for QW lasers showing a fast oscillation frequency $f_{\mathrm{FO}}$ that coincides with $f_{\text {ext }}$, the RPP state observed in this work has $f_{\mathrm{FO}}$ around $f_{\mathrm{RO}}=3 \mathrm{GHz}$ instead. This difference might be attributed to the fact the QD laser does not operate in the ultra short cavity regime for which $f_{e x t}>>f_{R O}$, but rather in a short delay configuration for which the relaxation oscillation frequency remains still pretty close to the external cavity frequency. In addition, it has to be stressed that the slow oscillation has a frequency $f_{\mathrm{SO}}=72 \mathrm{MHz}$ that is much lower than both $f_{\text {ext }}$ and $f_{\mathrm{RO}}$. Last but not the least, as compared to the RPP states reported in ${ }^{10}$ with less than 10 oscillations in each package, more than 40 oscillations are packed in the RPP states found in this GS laser. Figure 4 shows the evolution of $f_{S O}$ as a function of the external cavity frequency for two values of the bias current namely 1.5 times and 1.7 times the threshold respectively. Results show that there is a slight dependence of the slow oscillation with the bias current which is in agreement with prior works on the RPP regime in QW lasers ${ }^{11} .{ }^{12}$ For instance, for an external cavity frequency, experiments show that $f_{S O}$ increases by about $20 \mathrm{MHz}$ with the bias current. To the best of our knowledge, this RPP regime has never been observed so far in QD lasers.

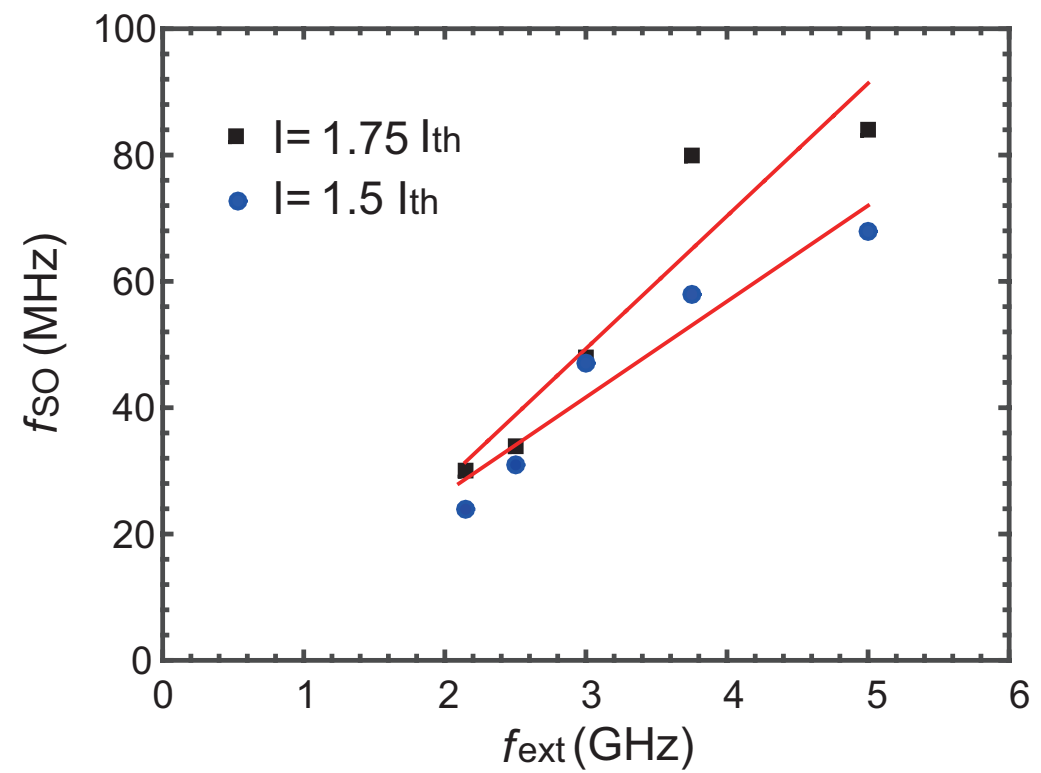

Figure 4. Evolution of the SO frequency $f_{S O}$ measured in the RPP regime for the GS laser at 1.5 times and 1.7 times the threshold respectively.

When $\xi_{\mathrm{f}}$ decreases to 0.731 , the laser becomes more stable and a $\mathrm{P}$ state oscillating at $f_{\mathrm{RO}}$ is found as shown in Figures 4(c) and 4(d) is found. Further increase of the external cavity length to the long cavity regime leads to frequency-locking regimes as reported elsewhere. ${ }^{13}$ Experimental results reveal that the GS laser is perfectly stable such that no chaotic state was found whatever the feedback strength level used in this setup. Besides, the GS laser remains under the steady-state condition providing the feedback strength does not exceed $30 \%$ which is already much larger compared to any typical reflection levels taking place in a transmission system ${ }^{13} \cdot{ }^{14}$

Figure 5 shows the time series and corresponding power spectra of the dynamical states from the ES laser still measured at 1.7 times the threshold. With $L_{\text {ext }}=20 \mathrm{~mm}$ and with a strong feedback of $\xi_{\mathrm{f}}=0.703$, a C state with irregular intensity modulation and a spectrum broadly elevated from the noise floor is shown in Figures $5(\mathrm{a})$ and 5(b). When $\xi_{\mathrm{f}}$ decreases to 0.686, a QCPP state is found and shown in Figures 5(c) and 5(d). Different from a RPP state that has a fast oscillation at $f_{\text {ext }},{ }^{10}$ the fast oscillation of this QCPP oscillates at $f_{\mathrm{RO}}$. The $f_{\mathrm{SO}}$ does not coincide with either $f_{\mathrm{RO}}$ or $f_{\mathrm{ext}}$ but with a frequency of $340 \mathrm{MHz}$ instead. Moreover, unlike the spectra of typical RPP states that have frequency components of only fast or slow oscillations and their corresponding harmonics and beats, the spectrum of the QCPP state preserves the broadband characteristics as present in the 
$\mathrm{C}$ state. When $\xi_{\mathrm{f}}$ further decreases to 0.48 , as shown in Figures $5(\mathrm{e})$ and $5(\mathrm{f})$, a $\mathrm{P}$ state stably oscillating at $f_{\mathrm{FO}}=4 \mathrm{GHz}$ is obtained. Finally, let us stress that in the long cavity regime, more complex dynamical states are observable in the ES laser hence leading to a complete route to chaos ${ }^{13}$ which which is fundamentally different than the dynamics displayed in the GS laser.
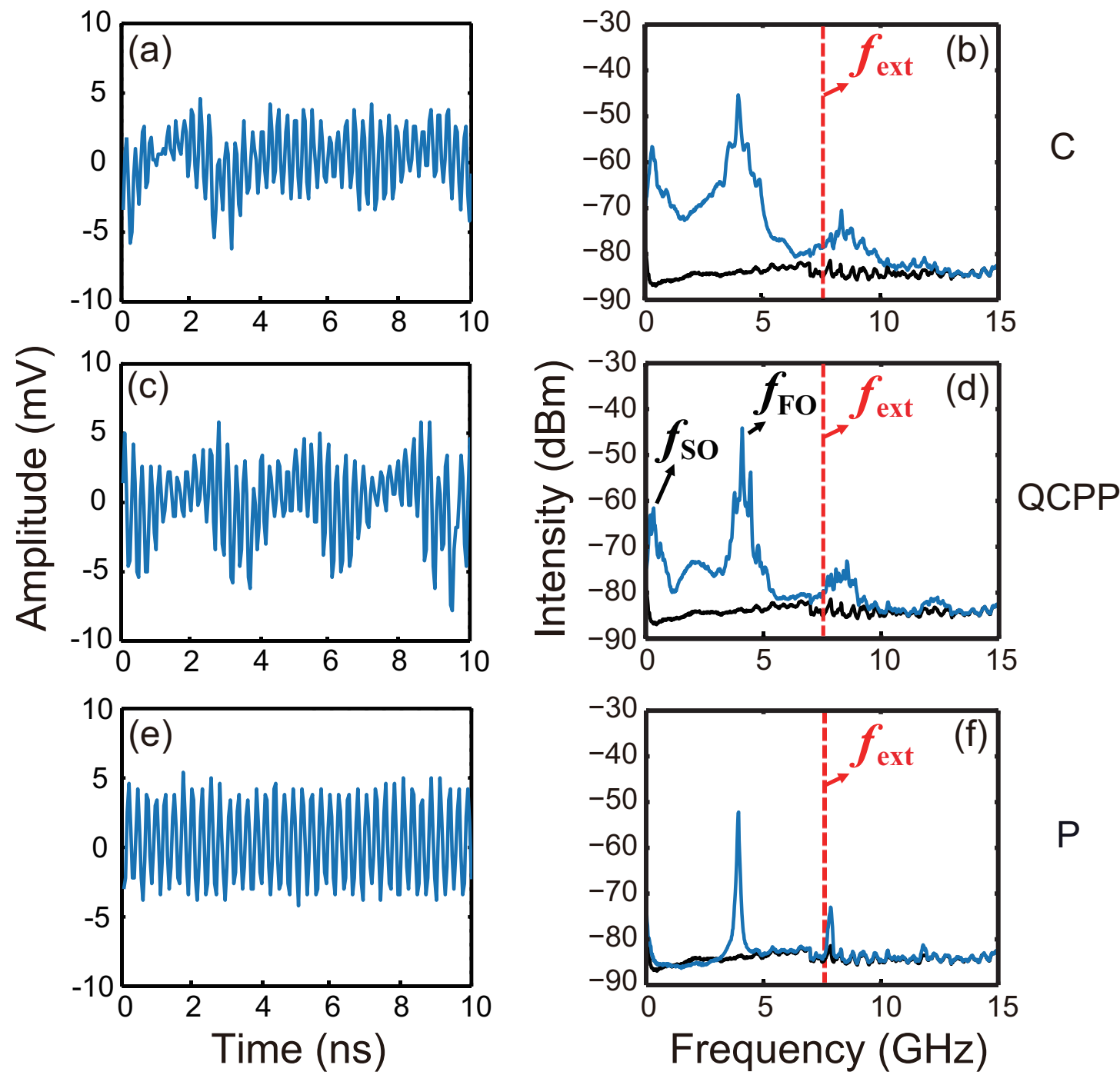

Figure 5. Time series and corresponding power spectra of the dynamical states measured at 1.7 times the threshold for the ES laser with $\left(\xi_{\mathrm{f}}, L_{\text {ext }}\right)=(\mathrm{a})(\mathrm{b})(0.703,20 \mathrm{~mm}),(\mathrm{c})(\mathrm{d})(0.686,20 \mathrm{~mm})$, (e)(f) $(0.48,20 \mathrm{~mm})$. Red-dashed lines depict the external cavity frequency $f_{\text {ext }}$ and black curves are the spectra without feedback for reference.

Figures 6 show $f_{\text {SO }}$ extracted from the RPP states of the GS laser and the QCPP states of the ES laser under different $f_{\text {ext }}$. As can be seen, while $f_{\mathrm{SO}}$ does not coincide exactly with either $f_{\mathrm{RO}}$ or $f_{\text {ext }}, f_{\mathrm{SO}}$ in both states increases linearly as $f_{\text {ext }}$ increases.

Compared to the GS laser which is in general stable and insensitive to feedback, the ES laser is more easily moving to complex dynamics. While a GS laser is of large importance for the development of isolator-free transmitters in short-reach networks, an ES laser on the other hand can be essential for applications taking advantages of chaos such as chaos lidars, chaos radars, and high-speed random number generations. ${ }^{15-18}$ From these results it is obvious that, although the GS and ES lasers have the same active medium, their response to the feedback are very different. Unlike the ES laser, the carrier dynamics of the GS laser involves transport, capture, and relaxation, leading to a larger damping rate that stabilizes the laser and prevents the development 


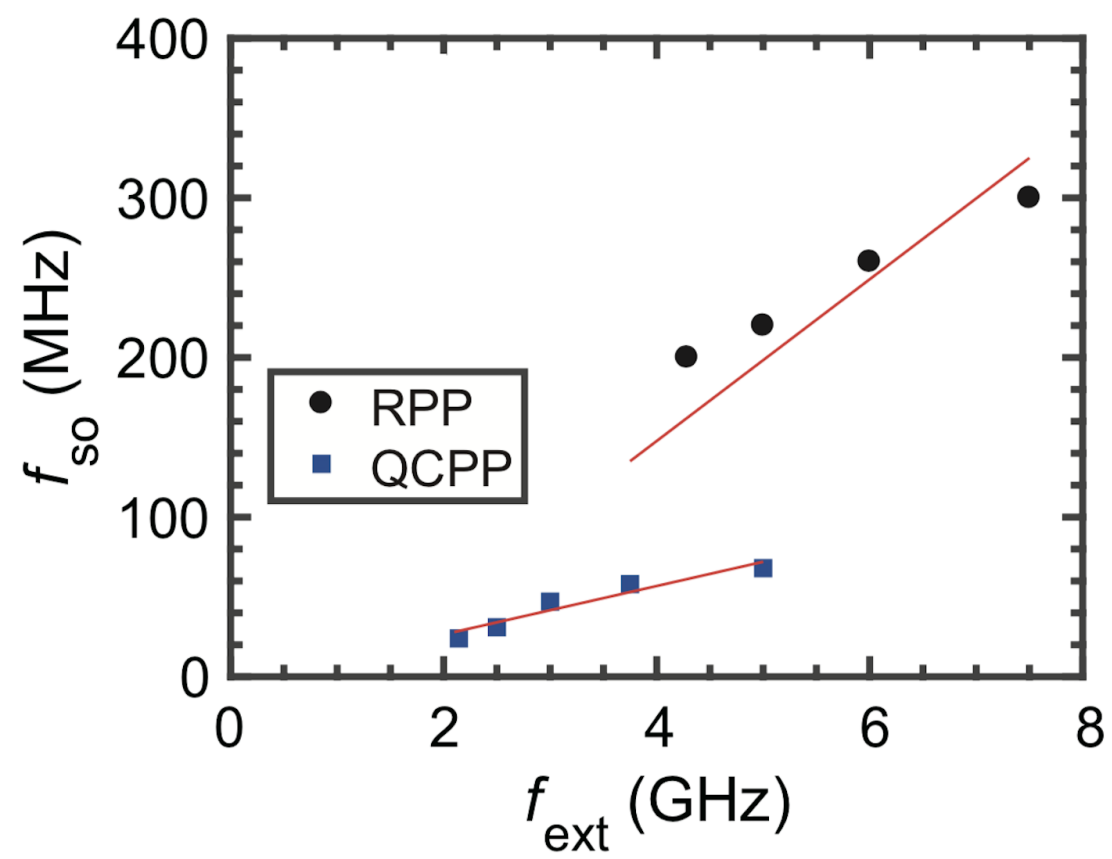

Figure 6. Slow oscillation frequencies $f_{\text {SO }}$ of the RPP and QCPP states under different external cavity frequencies $f_{\text {ext }}$ of both GS and ES lasers respectively. Red lines are the linear fits.

of complex dynamics. As reported in, ${ }^{9}$ the damping is as large as $18 \mathrm{GHz}$ for the GS laser whereas it is below 1 $\mathrm{GHz}$ for the ES one. Moreover, the ES laser has a stronger modal competition ${ }^{9}$ which also makes it easier to be driven into instabilities.

\section{CONCLUSIONS}

This work investigates for the first time the dynamical states and their spectral characteristics of optical feedback InAs/GaAs QD lasers emitting exclusively on single lasing states in the short cavity regime. Although the GS and ES QD lasers are made from the same active medium, their feedback dynamics are found not much alike. The GS laser is shown to be almost insensitive to feedback, especially at higher bias levels. No chaotic states was found which is of vital importance for the development of high-speed transmission links operating without isolator, in agreement with some earliest results. In contrast, the ES laser exhibits a plethora of complex dynamics including chaotic states, especially at higher bias levels, thus being useful for integrated self-pulsating devices, chaotic lidars and radars as well as high-speed random bit generations.

\section{ACKNOWLEDGMENTS}

This work was funded by Campus France (PhC Orchid No. 33721SC), the Institut Mines Télécom (IMT) through the Futurs \& Ruptures program and the Ministry of Science and Technology, Taiwan, under contracts MOST 105-2911-I-007-501 and 103-2112-M-007-019-MY3.

\section{REFERENCES}

[1] Kane, D. M. and Shore, K. A., [Unlocking Dynamical Diversity: Optical Feedback Effects on Semiconductor Lasers], Wiley (2005).

[2] Crowley, M. T., Naderi, N. A., Su, H., Grillot, F., and Lester, L. F., [GaAs-Based Quantum Dot Lasers, in Advances in Semiconductor Lasers], Academic Press (2012).

[3] Eisenstein, G. and Bimberg, D., [Green Photonics and Electronics], Springer (2017).

[4] Shchekin, O. and Deppe, D., "1.3 $\mu \mathrm{m}$ InAs quantum dot laser with $T_{0}=161^{\circ} \mathrm{K}$ from 0 to $80^{\circ} \mathrm{C}$," Appl. Phys. Lett. 80, 3277 (2002). 
[5] Luedge, K., [Nonlinear Laser Dynamics: From Quantum Dots to Cryptography], Wiley (2012).

[6] Arsenijević, D. and Bimberg, D., "Quantum-dot lasers for 35 Gbit/s pulse-amplitude modulation and 160 Gbit/s differential quadrature phase-shift keying," Proc. of SPIE 9892, 98920 (2016).

[7] Virte, M., Breuer, S., Sciamanna, M., and Panajotov, K., "Switching between ground and excited states by optical feedback in a quantum dot laser diode," Appl. Phys. Lett. 105, 121109 (2014).

[8] Sciamanna, M. and Shore, K. A., "Physics and applications of laser diode chaos," Nat. Photonics 9, 151 (2015).

[9] Huang, H., Arsenijević, D., Schires, K., Sadeev, T., Bimberg, D., and Grillot, F., "Multimode optical feedback dynamics of InAs/GaAs quantum-dot lasers emitting on different lasing states," AIP Advances 6(12), 125114 (2016).

[10] Heil, T., Fischer, I., Elsäßer, W., and Gavrielides, A., "Dynamics of semiconductor lasers subject to delayed optical feedback: The short cavity regime," Phys. Rev. Lett. 87(24), 243901 (2001).

[11] Sciamanna, M., Tabaka, A., Thienpont, H., and Panajotov, K., "Intensity behavior underlying pulse packages in semiconductor lasers that are subject to optical feedback," J. Opt. Soc. Am. B 22(4), 777 (2005).

[12] Tabaka, A., Panajotov, K., Veretennicoff, I., and Sciamanna, M., "Bifurcation study of regular pulse packages in laser diodes subject to optical feedback," Phys. Rev. E 70(3), 036211 (2004).

[13] Lin, L. C., Chen, C. Y., Huang, H., Arsenijevic, D., Bimberg, D., Grillot, F., and Lin, F. Y., "Comparison of optical feedback dynamics of InAs/GaAs quantum-dot lasers emitting solely on ground or excited states," Optics Lett. 43, 210 (2018).

[14] Huang, H., Lin, L. C., Chen, C. Y., Asenijevic, D., Bimberg, D., Lin, F. Y., and Grillot, F., "Multimode optical feedback dynamics in InAs/GaAs quantum dot lasers emitting exclusively on ground or excited states: transition from short- to long-delay regimes," Optics Express 26, 1743 (2018).

[15] Lin, F. Y. and Liu, J. M., "Chaotic lidar," IEEE J. Sel. Topics Quantum Electron. 10, 991 (2004).

[16] Cheng, C. H., Chen, C. Y., Chen, J. D., Pan, D. K., Ting, K. T., and Lin, F. Y., "3D pulsed chaos lidar system," Optics Express 26, 12230 (2018).

[17] Lin, F. Y. and Liu, J. M., "Chaotic radar using nonlinear laser dynamics," J. Quantum Electron. 40, 815 (2004).

[18] Takahashi, R., Akizawa, Y., Uchida, A., Harayama, T., Tsuzuki, K., Sunada, S., Arai, K., Yoshimura, K., and Davis, P., "Fast physical random bit generation with chaotic semiconductor lasers," Opt. Express 22(10), 11727 (2014). 